[24] Im Übrigen kann entgegen der nicht begründeten Auffassung des Berufungsgerichts nach den bisherigen Feststellungen nicht davon ausgegangen werden, dass der Verkäufer im Innenverhältnis allein für die Unterbringungs- und Behandlungskosten haftet. Hat sich der Verkäufer, was er im Prozess geltend gemacht hat, ebenso wie die K1. auf das Ankaufsgutachten verlassen, kann nicht ohne Weiteres angenommen werden, dass der Verkäufer für diese Kosten im Verhältnis zum Bekl. allein haftet. In diesem Fall könnte auch nicht ohne Weiteres angenommen werden, dass der Verkäufer deshalb im Innenverhältnis das alleinige oder überwiegende Verschulden trägt, weil er den Rücktritt nicht sofort akzeptiert hat.

[25] 3. Unbegründet sind die Bedenken des Berufungsgerichts, die Kl. müsse sich möglicherweise entgegenhalten lassen, sich durch den Vergleich der Ansprüche des Bekl. auf Herausgabe des Pferdes begeben zu haben, die im Falle eines gegen den Bekl. bestehenden Schadensersatzanspruches diesem zugestanden hätten. Dem allein auf Zahlung der Unterbringungs- und Behandlungskosten in Anspruch genommenen Bekl. steht kein Zug um Zug zu verwirklichender Anspruch auf Herausgabe des Pferdes zu. Dafür fehlt es an jeder Rechtsgrundlage.

[26] III. Der Senat kann in der Sache nicht selbst entscheiden. Das Berufungsurteil war daher aufzuheben und die Sache war zur Nachholung der erforderlichen Feststellungen an das Berufungsgericht zurückzuverweisen.

DOI: 10.1007/s00350-013-3536-8

\section{Anmerkung zu BGH, Urt. v. 22.12.2011 - VII ZR 7/11 (OLG Celle)}

\section{Jens Adolphsen und Robert Schenk}

Die Entscheidung des BGH bestätigt zunächst die mittlerweile gefestigte Ansicht der Einordnung der Kaufuntersuchung des Tierarztes als Werkvertrag mit der Folge, dass das Nichterkennen von Befunden Schadensersatz wegen Schlechtleistung nach sich zieht ${ }^{1}$. Vor Geltendmachung des Schadensersatzanspruches ist es nicht erforderlich, eine Frist zur Nacherfüllung zu setzen, weil dies unter Zugrundelegung normaler Umstände keinen Erfolg haben kann².

Klarheit bringt die Entscheidung für die Voraussetzungen der Gesamtschuld. Zwischen dem Tierarzt, der die Kaufuntersuchung fehlerhaft vornimmt, und dem Verkäufer, der ein mangelhaftes Tier liefert, ist ein Gesamtschuldverhältnis für Mangelfolgeschäden anzunehmen, da insoweit ein identisches Leistungsinteresse vorliegt und die gegenüber dem Käufer bestehenden Verpflichtungen der Schuldner gleichstufig nebeneinander stehen ${ }^{3}$. Der BGH hat anders lautende Ansichten der OLGe ${ }^{4}$, wonach eine Gesamtschuldnerschaft mangels Gleichstufigkeit nicht bestehe, zurückgewiesen und zu Recht betont, dass insoweit allein die Verpflichtung zur Befriedigung inhaltsgleicher Gläubigerinteressen entscheidend $\operatorname{sei}^{5}$. Im Ergebnis ist die Haftung des Veterinärs im Vergleich zur Haftung des Verkäufers jedenfalls nicht subsidiär. Bringt das Urteil des BGH zumindest im Ergebnis Klarheit über das Bestehen gesamtschuldnerischer Haftung zwischen Verkäufer und Tierarzt für Mangelfolgeschäden, bedeutet dies zugleich, dass der die Kaufuntersuchung durchführende Veterinär zukünftig vermehrt haftungsrechtlicher Inanspruchnahme zum einen durch den Tierkäufer, aber auch durch den Regress neh-

Prof. Dr. iur. Jens Adolphsen und Wiss. Mitarb. Robert Schenk, Professur für Bürgerliches Recht, nationales und internationales Zivilverfahrensrecht und Sportrecht, Justus-Liebig-Universität, Licher Straße 76, 35394 Gießen, Deutschland menden gesamtschuldnerisch haftenden Verkäufer, zu dem möglicherweise zuvor keinerlei vertragliche Verbindung bestand, ausgesetzt ist. Für Berufshaftpflichtversicherer der Tierärzte könnte dieses Urteil Anlass zur Beitragserhöhung geben, da mit zunehmender gerichtlicher Inanspruchnahme ihrer Versicherten zu rechnen ist.

In Bezug auf den abgeschlossenen Vergleich geht der BGH im Ergebnis zu Recht von einer Einzelwirkung aus. $\mathrm{Ob}$ ein Vergleich Gesamtwirkung entfaltet, ist durch Vertragsauslegung zu ermitteln. Gesamtwirkung ist anzunehmen, wenn aus dem Vergleich ausdrücklich oder aus den Umständen hervorgeht, dass der Gläubiger den Willen hatte, gegenüber dem nicht am Vergleich beteiligten Gesamtschuldner auf weitergehende Ansprüche zu verzichten. Sofern keine Umstände ersichtlich sind, dass Gläubiger und Gesamtschuldner den nicht beteiligten Gesamtschuldner freistellen wollen, kann eine Gesamtwirkung oder allenfalls beschränkte Gesamtwirkung nicht angenommen werden ${ }^{6}$. Zielt der Gläubiger jedoch darauf $a b$, den den Vergleich schließenden Schuldner endgültig freizustellen, was i.d. R. auch für diesen von größtem Interesse ist, so ist die Vereinbarung einer beschränkten Gesamtwirkung die wohl interessengerechte Lösung, da der Verkäufer nicht durch Regress des am Vergleich nicht beteiligten Gesamtschuldners in Anspruch genommen werden kann. Auch der Tierarzt wäre nicht mehr der vollen Haftung ausgesetzt und der Käufer kann zumindest noch die nicht vom Vergleich gedeckten Ansprüche, für die der Verkäufer nicht in Regress genommen werden kann, gegenüber dem Tierarzt geltend machen. Die Überlegung geht dahin, künftig nicht allzu hohe Anforderungen an die Annahme einer beschränkten Gesamtwirkung zu stellen?

1) Vgl. BGHZ 87, 239; OLG Hamm - 24 U 134/94 -, NJW-RR 1996, 736

2) Adolphsen, in: Terbille (Hrsg.), Münchener Anwaltshandbuch Medizinrecht, 1. Aufl. 2009, \$12, Rdnr. 247.

3) So auch Bydlinski, in: MüKo/BGB, Bd. 2, 6. Aufl. 2012, $\ 421$, Rdnrn. 5 und $12 \mathrm{ff}$.

4) OLG Karlsruhe - 11 U 16/97 -, NJW-RR 1998, 601; OLG Schleswig - 13 U 8/10 -, RdL 2011, 208.

5) So auch BGH - VII ZR 126/02 -, NJW 2003, 2980; Bydlinski, in: MüKo/BGB, Bd. 2, 6. Aufl. 2012, $\ 421$, Rdnrn. 5 und $12 \mathrm{ff}$.; Grüneberg, in: Palandt, BGB, 63. Aufl. 2013, $\$ 421$, Rdnrn. $6 \mathrm{f}$

6) BGH - XI ZR 39/99 -, NJW 2000, 1942; BGH - I ZR 249/01 -, NJW-RR 2005, 34

7) So bereits Looschelders, JA 2012, 301

\section{Hypothetische Einwilligung bei Anwendung einer "Neulandmethode“}

StGB $§ 224,227,263$

1. Zu den Voraussetzungen einer hypothetischen Einwilligung beim ärztlichen Heileingriff.

2. Zum Täuschungsvorsatz als Voraussetzung für eine Strafbarkeit wegen Abrechnungsbetruges. (Leitsätze des Bearbeiters)

BGH, Urt. v. 20.2.2013 - 1 StR 320/12 (LG Kempten)

Problemstellung: Mit nachstehendem Urteil hat der 1. Strafsenat des BGH die schon in der früheren Rechtsprechung aufgestellten Grundsätze zur rechtlichen Wirkung einer ,hypothetischen Einwilligung“ nochmals

Bearbeitet von Rechtsanwalt Jürgen Pauly,

Fachanwalt für Strafrecht,

Wolfsgangstraße 92, 60322 Frankfurt a. M., Deutschland 